\title{
DEVELOPMENT TOOLS OF THE INTELLECTUAL SELF-ORGANIZED SYSTEMS OF AUTOMATIC CONTROL
}

\author{
M.F. Stepanov ${ }^{1}$, A.M. Stepanov², M.A. Pakhomov ${ }^{1}$, A.R. Salikhova ${ }^{1}$, \\ L.S. Mikhaylova ${ }^{3}$ \\ ${ }^{1}$ Yury Gagarin State Technical University of Saratov, Saratov, Russia \\ ${ }^{2}$ Institute of Problems of Exact Mechanics and Control of the Russian Academy of Science \\ ${ }^{3}$ Electrosteel Polytechnical Institute of the Moscow State Machine-Bbuilding University, Moscow, \\ Russia
}

\begin{abstract}
Questions of automation of creation of systems of the decentralized control of non-stationary plants by artificial intelligence methods are considered. As one of solutions of the specified problem the union of the intellectual self-organized systems of automatic control and neurocontrol is offered: 1) synthesis of the control law with use of means of the intellectual selforganized systems of automatic control (ISSAC); 2) implementation of the synthesized control law with use of the artificial neural network (ANN). The offered approach includes parallelization of control processes, identifications (creating of the emulator), synthesis of the control law and, at last, creating of the neurocontroller according to parameters of the new control law. With respect thereto the generalized scheme of implementation of ISSAC in the form of set of the calculator on the microprocessor and the blocks constructed on FPGA in which the plants emulator and the neurocontroller are realized is offered.
\end{abstract}

Keywords: Information technologies, intellectual systems, automatic control, self-organization.

Citations: Stepanov MF, Stepanov AM, Pakhomov MA, Salikhova AP, Mikhailova LS. Development tools of the intellectual self-organized systems of automatic control. CEUR Workshop Proceedings, 2016; 1638: 674-680. DOI: 10.18287/1613-0073-2016-1638-674-680

\section{Introduction}

Progress of ADP equipment brought high-performance tools of data handling and control. Information technologies provided methods of effective processing of large volumes of data. Together they removed boundary of area in which perhaps detail computation and planning, but didn't remove it absolutely. On the other hand, in cy- 
bernetics (science about control) methods of synthesis and the analysis of laws of control of harder and harder control plants are developed.

The most important concepts of the modern theory of control are the self-adjustment and invariance. Invariance - property of automatic systems to resist to perturbations. A self-adjustment - means by means of which invariance as the purpose is reached. For complex control tasks it is inherent variability not only perturbations, plants parameters, but also purposes of control. In such cases transition to the self-organized systems is expedient. Their difference from the self-setting-up systems is ability to change automatically not only parameters, but also structure of the law of control. In a general view appearance of additional channels of obtaining information and control (fig. 1) for this purpose is necessary. However and there are unresolved problems. In particular, there is a problem of criteria of self-organization.

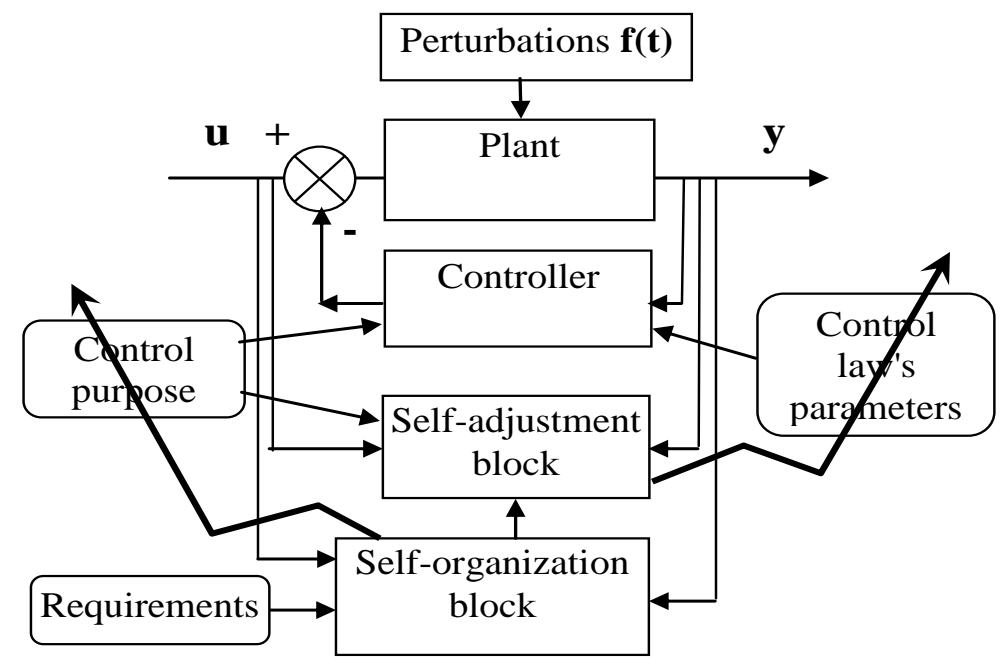

Fig. 1. Structure of the self-organized systems

\section{The intellectual self-organized systems of automatic control}

Conceptually the intellectual self-organized systems of automatic control (ISSAC) [1], as a distinctive feature, contain intellectual system of synthesis of the law of control on the given purpose of control, and also means of formation of the purpose of control on the basis of information on the environment of functioning and purposes of system's functioning (see fig. 2). 


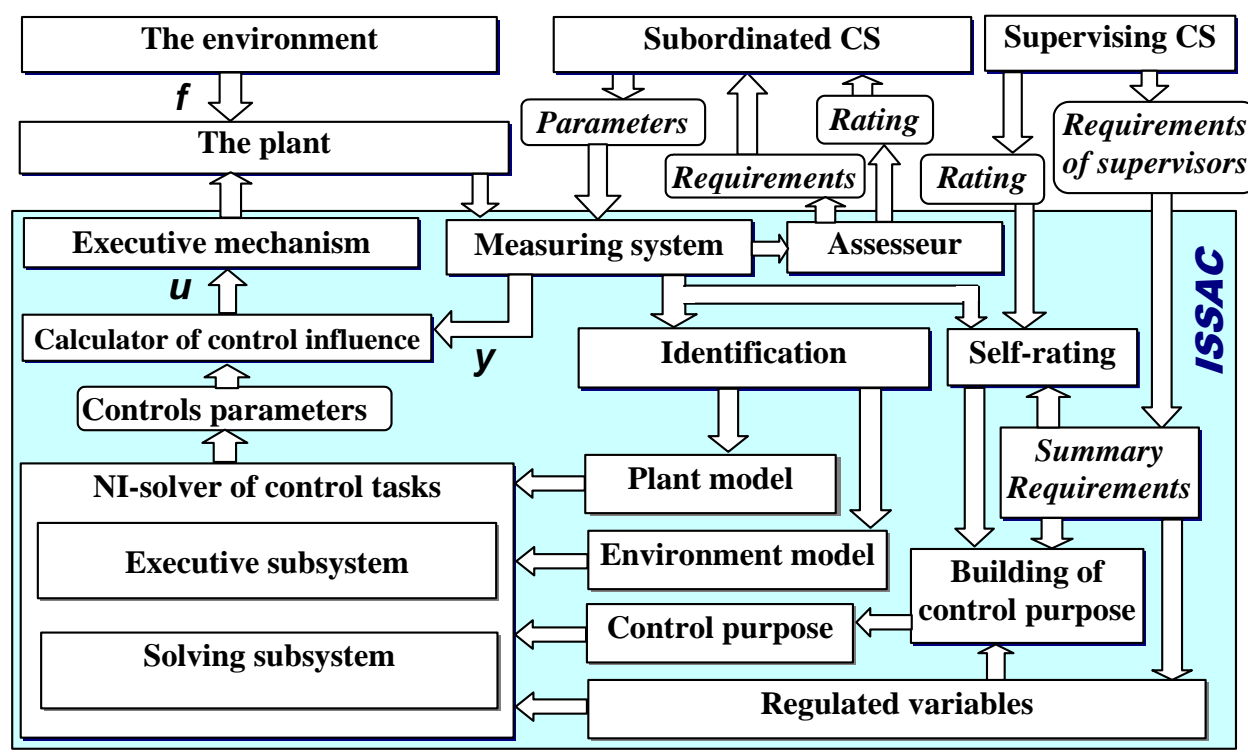

Fig.2. The diagram of the intellectual self-organized system of automatic control

\section{Criteria of self-organization}

The control task definition includes:

Control plant:

$$
y=P\left(x, u, f, v, M_{o}\right)
$$

Perturbations: $F=\left\{f|| f_{i} \mid \leq f_{i}^{*}, i=(\overline{1, \mu})\right\}, V=\left\{v|| v_{i} \mid \leq v_{i}^{*}, i=(\overline{1, v})\right\}$

Controlled variable:

$$
\theta=N x, \quad \theta \in R^{\chi}
$$

Control purpose:

$$
\Psi=\left\{\Psi_{1}, \ldots \Psi_{k}, \ldots, \Psi_{n_{\Psi}}\right\}
$$

Control:

$$
u(t)=U\left(t, y, M_{U}\right)
$$

Control synthesis:

$$
M_{U}=S\left(M_{O}, \Psi, F, V, Э\right)
$$

Self-organization:

$$
Q=Q_{\ni}\left(M_{O}, N, F, V, \Psi, \ni\right)
$$

Traditional forms of the purpose of control, for example, requirements to accuracy (8) don't allow to organize effectively an assessment of need of switching one of the modes of self-organization. 
$\Psi_{1}(\theta)=\left\{\begin{array}{l}1, \theta_{y c m} \leq Q_{1} \\ 0, \theta_{y c m}>Q_{1}\end{array}, \theta_{y c m}=\lim _{t \rightarrow \infty} \theta(t)\right.$

Therefore it is necessary to enter the modified forms of criteria of accuracy and quality of control (9) - (10).

$$
\begin{aligned}
& \Psi_{1}(\theta)= \begin{cases}\alpha, & \theta_{y c m}<\beta Q_{1}, \alpha>1, \beta<1 \\
0, & \theta_{y c m}=Q_{1} \\
-\alpha, & \theta_{y c m}>Q_{1}\end{cases} \\
& \Psi_{k}\left(\theta, u, t, Q_{k}\right)=\left\{\begin{array}{lr}
\alpha, & J=J\left(\theta, u, t, Q_{k}\right)<J^{*} \\
0, & J=J\left(\theta, u, t, Q_{k}\right)=J^{*} \\
-\alpha, & J=J\left(\theta, u, t, Q_{k}\right)>J^{*}
\end{array}\right. \\
& J\left(\theta, u, t, Q_{k}\right)=\int_{0}^{\infty}\left(\theta^{T} Q_{k, \theta} \theta+u^{T} Q_{k, u} u\right) d t
\end{aligned}
$$

The generalized assessment received the name "emotions" [2] in memory of physiological roots of the P. K. Anokhin's theory of functional system [3] as first, full completed model of cybernetic system. To qualities control of difficult multilayer decentralized systems we uses assessment for subsystems (11). It allows controlling underlying subsystems by means of use of mechanisms of their self-organization.

$$
\ni=\sum_{k=1}^{n_{\psi}} \alpha_{k} \Psi_{k}\left(\theta, u, t, Q_{k}\right)+\sum_{k=1}^{\hat{n}_{\psi}} \hat{\Psi}_{k}\left(\hat{\theta}, t \hat{Q}_{k}\right) \in \Im^{*}, \sum_{k=1}^{n_{\Psi}} \alpha_{k}=1, \Im^{*}=\left\{\vartheta_{-}, \ni^{-}\right\}
$$

In case of changes of a control object and the purposes of control (13) the choice of procedure of synthesis of the law of control is difficult. Attraction of methods of artificial intelligence allows solving tasks [1]. But it requires formalization of knowledge of the theory of automatic control (TAC) [4] as a triad

$$
M_{O}=\langle\Pi, Д, O\rangle
$$

where $\Pi=\left\{n_{1}, \ldots, n_{\pi}\right\}$ - a set of the formalized generalizations of models of the components of the automatic control system (ACS) called by "predmets", possessing properties $\quad p_{j} \in P_{i} \subset \wp=\{\rho \mid \rho \in\{$ true $\mid$ false $\}\} \quad, \quad$ characteristics $h_{j} \in H_{i} \subseteq \aleph=\left\{\chi_{k} \mid \chi_{k} \in C^{N_{k}}\right\}$, where C - a set of complex numbers; forms of mathematical models $m_{j} \in \Phi_{i} \subset \mathfrak{I}=\left\{\mu_{1}, \ldots, \mu_{\tau}\right\}$; 
$Д=\{d \mid d: \wp \cup \aleph \cup \mathfrak{I} \rightarrow \wp \cup \aleph \cup \mathfrak{I} \cup \mathrm{O}\}$ - set of the operations (actions) performed over predmets, their properties and characteristics; $\mathrm{O}=\{o \mid o: \wp \cup \mathfrak{} \cup \mathfrak{I} \rightarrow\{$ true $\mid$ false $\}\}$ - set of the relations (predicates) defined on sets of predmets, their properties and characteristics. Actions $d_{i}=\left\langle y_{i}, \partial_{i}, r_{i}, q_{i}\right\rangle \in Д$ and the relations $o_{i}=\left\langle y_{i}, \partial_{i}, r_{i}\right\rangle \in O$ are unambiguous identified by the attributes: $y_{i} \in \wp \cup \mathrm{O}$ - applicability conditions, $\partial_{i} \in \wp \cup \mathfrak{\cup}$ source data, $r_{i} \in \wp \cup \aleph \cup \cup \mathfrak{T} \cup \mathrm{O}$ - results of action, $q_{i} \in \mathrm{O}$ - requirements to results of action, $p_{i} \in\{$ true $\mid$ false $\}$ - value of the relation.

The model is used in intellectual system of synthesis of the law of control for creation of the most adequate procedure of synthesis. Simulation of such systems is a complex challenge. The system of Modeling of the Intellectual Self-organized Systems - MISS is for this purpose developed [5]-[6].

We research possibilities ISSAC for control of non-stationary plant (see fig. 3). Let the plant of control is described by the following equations:

$$
\begin{aligned}
& \dot{x}=(A+\Delta A) x+u^{*}+M f \\
& x \in R^{n}, u \in R^{n}, f \in R^{\mu} \\
& \Delta A=\left\{\begin{array}{l}
{[0]_{n}, 0 \leq t<t_{0}} \\
\Delta A=d A \times \sin \left(\omega \times\left(t-t_{0}\right)\right), \forall t \geq t_{0}
\end{array}\right. \\
& f=\left\{\begin{array}{l}
f_{0}, \quad 0 \leq t<t_{S} \\
f_{0}+f_{m} \times \sin \left(\omega_{f} \times\left(t-t_{S}\right)\right), \quad \forall t \geq t_{S}
\end{array}\right.
\end{aligned}
$$

were $t_{0}=4$ - the moment of the beginning of a modification of plant's model; $\omega-$ frequency of a modification of plant's model; $f_{0}=1,0$ - magnitude of stepping component exterior perturbation; $f_{m}=0,25$ - amplitude of sine waves of the exterior perturbation; $\omega_{f}$ - frequency of sine wave of the exterior perturbation; $t_{S}=5-$ the moment of inclusion of sine wave of the exterior perturbation; $[0]_{n}-$ zero matrix $n \times n$.

The purpose of control is set as requirements on the statically errors of controlled variables:

$$
\theta=N x, \theta \in R^{\chi}, N=\left[\begin{array}{lll}
1 & 0 & 0
\end{array}\right],\left|\theta_{\mathrm{st}_{i}}\right| \leq \theta_{\mathrm{st}_{i}}^{*}, \theta_{\mathrm{st}^{*}}^{*}, \theta_{\mathrm{st}} \in R^{\chi}, \theta_{\mathrm{st}_{1}}^{*}=0,5
$$

at presence of stepping exterior perturbations $f_{0}=0,5$. The initial law of control was synthesized counting upon stepping exterior perturbation $f_{0}=0,5$. Therefore with perturbation $f_{0}=1,0$ of the requirement to exactitude of regulating at the disconnected self-organizing are not fulfilled even for stationary plant (a curve 1 on fig. 4). Inclusion of self-organizing in an instant $t_{C}=10,0$ with periodicity in 1 second and 
with a velocity of self-organizing $\mathbf{0 , 1 7}$ eliminates a problem, ensuring a required exactitude of regulating (a curve 2). The transient for non-stationary plant by control without self-organizing is viewed as curve 3. Inclusion of self-organizing with the same parameters ensures a required exactitude of regulating and for non-stationary plant (a curve 4).

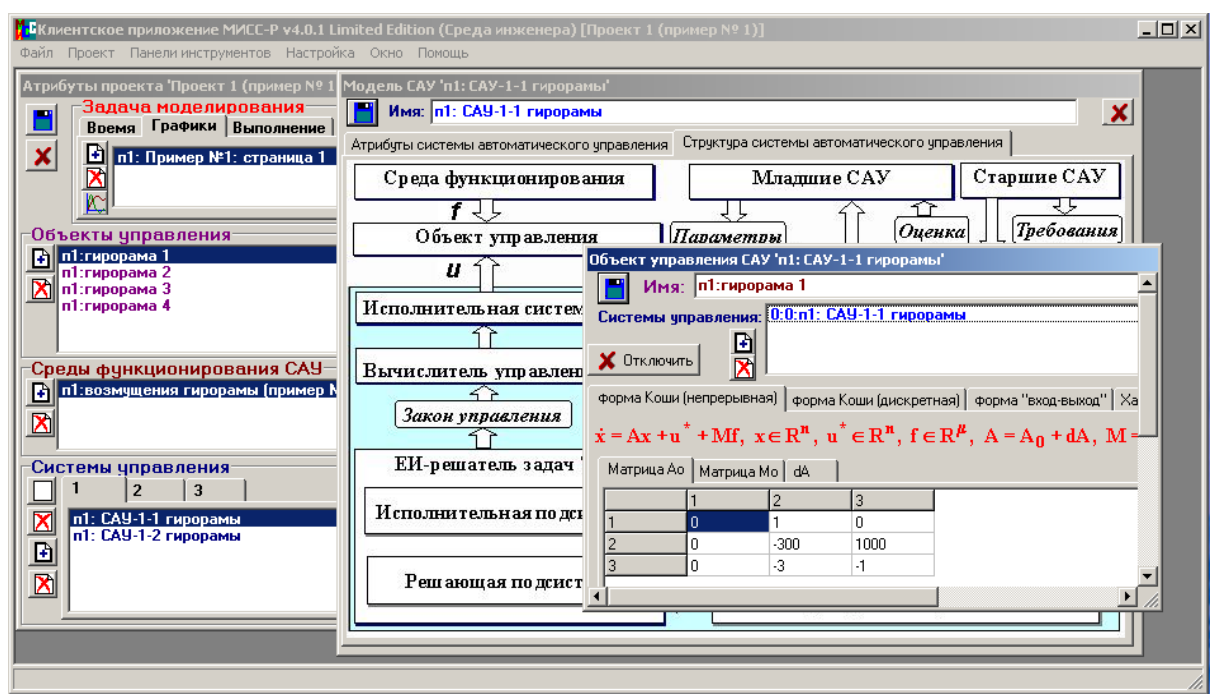

Fig. 3. Attributes of the simulation project

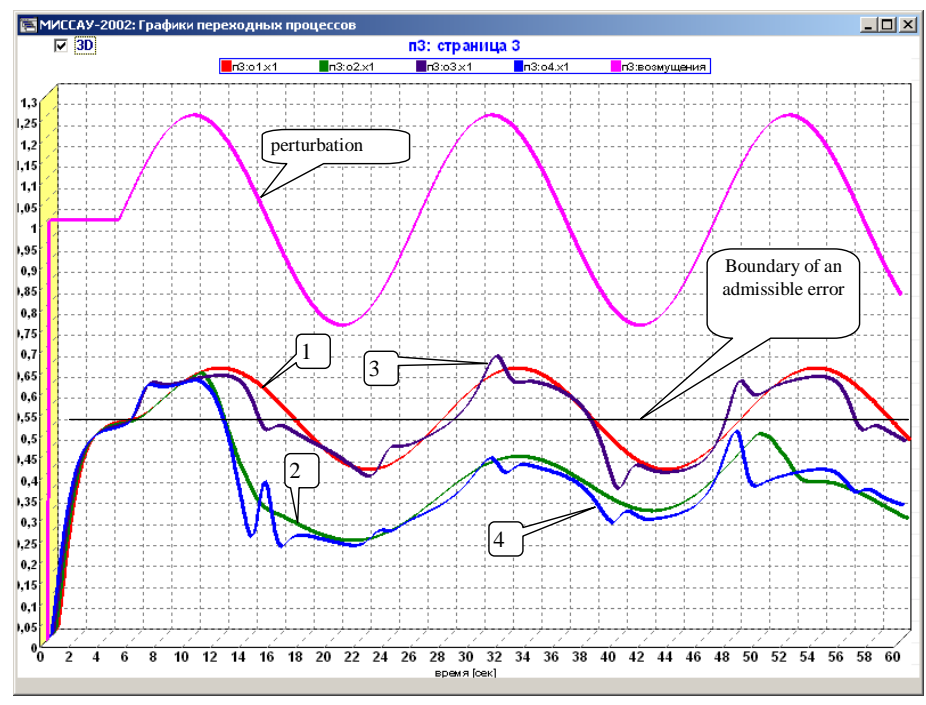

Fig. 4. Control of non-stationary plant 


\section{Conclusion}

The conducted researches showed operability of the offered approach in conditions of existence of not stationary of a plant when the stabilizing controller doesn't provide execution of the given requirements.

\section{Acknowledgements}

Work is performed with assistance of the Russian fund of basic researches (project 15-07-99684-a).

\section{References}

1. Stepanov MF. Intellectual self-organizing systems of automatic control. Saratov: Saratov state technical university, 2002. [in Russian]

2. Stepanov MF. The planning artificial neural networks in the self-organized intellectual control systems. Reports of the Russian academy of natural sciences. Volga region interregional office, 1999; 1: 73-99. [in Russian]

3. Anokhin PK, Shumilina AI, Anokhina AP and etc. Functional system as a basis of integration of nervous processes in embryogenesis. Proceedings $\mathrm{V}$ of congress of physiologists of the USSR, 1937: 148-156. [in Russian[

4. Alexandrov AG. Methods of creation of systems of automatic control. M.: Publishing house of physical and mathematical literature, 2008. [in Russian]

5. Stepanov MF, Stepanov AM. Application of planning artificial neural networks in solver of tasks of intellectual self-organizing automatic-control systems. Journal of Chaotic Modeling and Simulation: International Journal of Nonlinear Science, 2013; 1: 67-74.

6. Stepanov MF, Stepanov AM. Intellectual self-organizing control system with the calculator of the operating influence on the basis of automatically designed artificial neural network. The Bulletin of the Saratov state technical university, 2010; 4(51); Release 3: 126128. [in Russian] 